\title{
Precise Model-Free Spline-Based Approach for Magnetic Field Mapping
}

\author{
Federico Ongaro $^{1}$, Christoff M. Heunis ${ }^{1}$, and Sarthak Misra ${ }^{1,2}$ \\ ${ }^{1}$ Department of Biomechanical Engineering, University of Twente, The Netherlands. \\ ${ }^{2}$ Department of Biomedical Engineering, University of Groningen and University Medical Centre Groningen, The Netherlands.
}

\begin{abstract}
Untethered magnetic manipulation has found applications in a rapidly increasing number of fields, ranging from minimally invasive surgery to assembly of industrial micro electromechanical systems. Despite this relevance, present-day literature on precise magnetic mapping is sparse, especially for magnetic fields affected by external disturbances. In this work, we address this deficiency by introducing a model-free mapping technique. Remarkably, the presented spline-based approach is capable of addressing the presence of inhomogeneous static disturbances and the mapping of non-azimuthally symmetric electromagnets. The work is validated with the mapping of nine metal-core electromagnets in the presence of inhomogeneous static disturbances. A grid of 5120 measurements is collected, by a custom-programed robotic arm, and used for mapping. Further, the values predicted by the approach are compared against 3430 independent field measurements, obtaining an $R^{2}$ value of 0.9884 and maximum relative errors of $7 \%$. Overall, this spline-based approach provides a flexible technique for the precise mapping of electromagnetic fields and gradients even when, for reasons regarding coil-shape or disturbances, the electromagnetic field does not present any axial symmetry.
\end{abstract}

Index Terms-Electromagnetics, Magnetic actuators, Magnetic levitation, Numerical methods

\section{INTRODUCTION}

In recent years, electromagnetic manipulation has gained tremendous relevance for wireless actuation. The flexible and untethered nature of electromagnetic waves has made them a recurrent choice for the manipulation of a plethora of devices ranging from tethered to untethered, from active to passive, and from nano to macro scale, with applications in medical, chemical, biological, and industrial environments [1]-[10].

In particular, homogeneous magnetic fields are often used in these applications, allowing to control devices with up to 8 Degrees Of Freedom (DOFs) [11]. However, such fields are often generated by air-core electromagnets. Hence, they are subject to strong power constraints as the required workspace or field strength increase. Alternatively, researchers have proposed the use of inhomogeneous fields, as they offer both more DOFs and represent a more power-efficient solution [12].

Yet inhomogeneous fields come at the cost of a higher complexity and lower adherence to simple models. Consequently, commonly used mapping techniques - based on simple interpolations or first order approximations - fail to provide an accurate estimation of the fields and gradients used for inhomogeneous magnetic manipulation.

Notwithstanding the importance of precise mapping techniques, present-day literature on the subject is sparse. Remarkably, Petruska et al. recently presented a model-based approach for precise electromagnetic mapping [13]. Despite the considerable significance of such work, their approach can only be used with azimuthally symmetric electromagnets, has a lower bound on the number of measurements, and is not able to address the presence of external inhomogeneous static disturbances.

In this study, we investigate the development of a modelfree mapping technique. This novel approach is inspired by tensor product Basis Splines (B-Splines), which due to their properties, are the cornerstone of several engineering and computer graphics techniques [14]-[17]. The developed technique offers a model-free approach capable of precise mapping of electromagnetic fields and gradients, even when these are affected by static disturbances or are generated by non-symmetric electromagnets. Additionally, the algorithm can be applied to any number of measurements, enforces Maxwell's equation, and presents no lower or upper bound on the number of measurements. Moreover, the effectiveness of the technique is validated using an electromagnetic testbed (Fig. 1).

\section{B-SPLINES}

The presented work is based on the theory of B-Splines. While the basic theory of B-Splines is well known, we choose to give a brief summary of the used concepts and notations to ensure thorough understanding; for further details, we refer the reader to relevant literature [18], [19].

\section{A. Parametric B-Splines}

A B-Spline $(f)$ of non-negative degree $d \in \mathbb{N}$ is a parametric curve in an $\alpha$-dimensional space constructed from $n \in \mathbb{N}$ control points $\left(\mathbf{c}_{i}\right)_{i=1}^{n} \in \mathbb{R}^{\alpha}$ and a nondecreasing sequence of $n+d+1$ knots $\left(t_{i}\right)_{i=1}^{n+d+1} \in \mathbb{R}$ according to

$$
f(x)=\sum_{i=1}^{n} \mathbf{c}_{i} N_{i, d}(x)
$$

where $x \in \mathbb{R}$ is the scalar parametrization variable, and $N_{i, d}$ are the basis functions, recursively defined as follows:

$$
N_{i, d}(x)=\frac{x-t_{i}}{t_{i+d}-t_{i}} N_{i, d-1}(x)+\frac{t_{i+1+d}-x}{t_{i+1+d}-t_{i+1}} N_{i+1, d-1}(x)
$$

where:

$$
N_{i, 0}(x)= \begin{cases}1, & \text { if } t_{i} \leq x<t_{i+1} \\ 0, & \text { otherwise }\end{cases}
$$




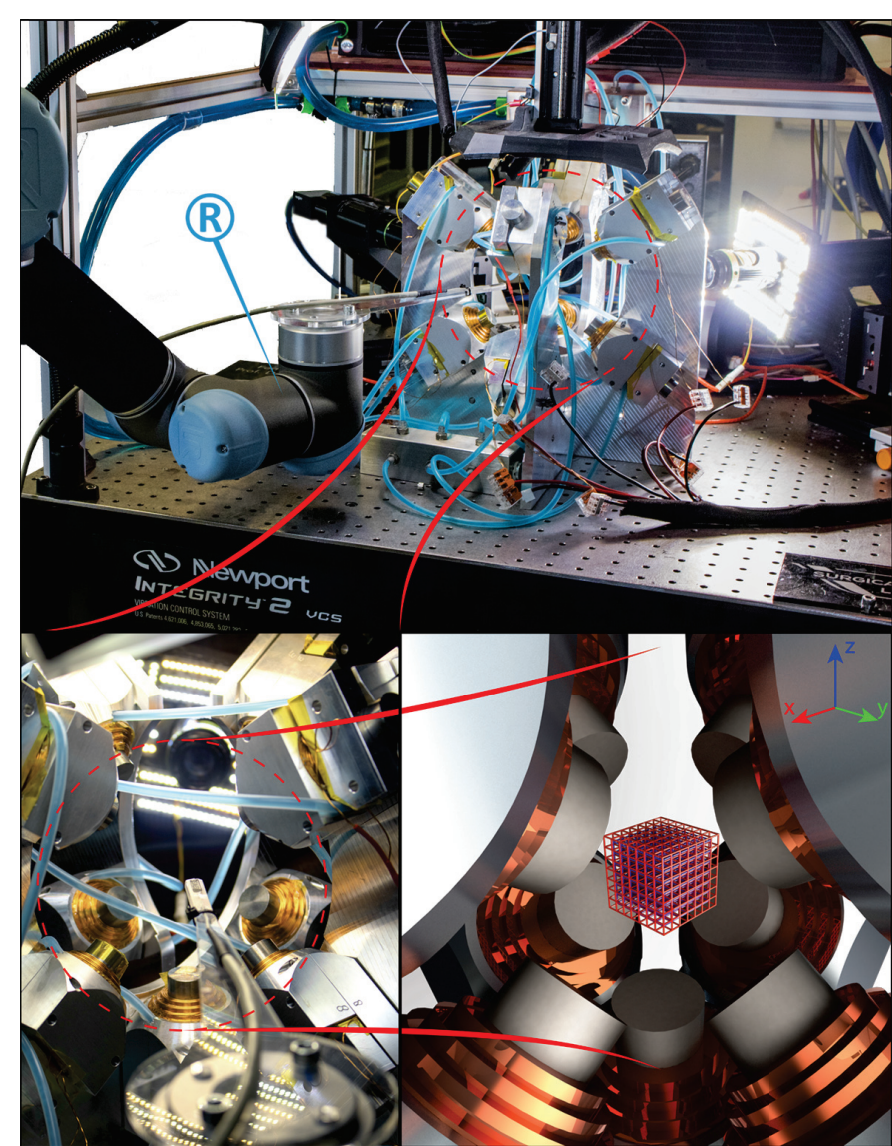

Fig. 1: Top: The setup used for the experimental validation of the model-free spline-based technique. The nine metal-core electromagnets of the electromagnetic setup generate an electromagnetic field that is measured by the calibrated three axis teslameter. In order to guarantee precise positioning the teslameter probe is attached to the end effector of a 6-DOF robotic arm R that places it in the desired position. Bottom-Left: Inside the workspace the custom-coded tracking system allows to improve the positioning of the probe. BottomRight: Graphical representation of the $8 \times 8 \times 8$ (red) and $7 \times 7 \times 7$ (blue) grids used for training and validation, respectively. A total of 8550 measurements were collected in the $14 \times 14 \times 14 \mathrm{~mm}^{3}$ workspace. The reference frame is oriented as shown, while its origin is in the center of the workspace.

It is worth noting that, due to the formulation of B-Splines, if $x \in\left[t_{i}, t_{i+1}\right)$ then $f(x)$ will belong to the convex hull of $\left(\mathbf{c}_{j}\right)_{j=i-d}^{i}$. Further, if $t_{i}<t_{i+1}$ the B-Spline is $C^{d-1}$ continuous [18], [19]. Finally, For notational simplicity, we will denote $N_{i, d}$ as $N_{i}$ for B-splines of known (or set) degree.

\section{B. Multi-dimensional Tensor Product B-Splines}

A B-Spline curve may have multi-dimensional co-domains, however its input is always a single scalar value. This can be an issue when a B-Spline function has to operate in a multivariable environment. Tensor products can be used to obtain B-Splines with multi-dimensional domains.

For instance, assume two positive integers $\left(d_{1}, d_{2}\right)$ and two knot vectors $\left(\sigma_{1}\right.$, and $\left.\sigma_{2}\right)$ yielding basis function spaces $\left(\mathbb{S}_{k}, k=1,2\right)$

$$
\begin{gathered}
\mathbb{S}_{1}=\mathbb{S}_{d_{1}, \sigma_{1}}=\operatorname{span}\left\{N_{1}, \ldots, N_{n_{1}}\right\} \\
\mathbb{S}_{2}=\mathbb{S}_{d_{2}, \sigma_{2}}=\operatorname{span}\left\{M_{1}, \ldots, M_{n_{2}}\right\} .
\end{gathered}
$$

The tensor product $\left(\mathbb{S}_{1} \otimes \mathbb{S}_{2}\right)$ of these spaces can be expressed as [19]

$$
f(x, y)=\sum_{i=1}^{n_{1}} \sum_{j=1}^{n_{2}} \mathbf{c}_{i, j} N_{i}(x) M_{j}(y)
$$

where $\left(\mathbf{c}_{i, j}\right)_{i, j=1}^{n_{1}, n_{2}}$ is the set of control points. Using such formulation, (5) allows to have a B-Spline behavior with a multidimensional domain. Clearly, this procedure can be iterated to obtain multi-variable B-Splines of any degree or dimension.

\section{Tensor Product B-Splines For MAGNETIC 3D MAPPING}

Specifically, in this work we will be using tricubic B-Splines (three-variable tensor product B-Splines with $d=3$ ) to calibrate the electromagnetic field. The general formulation of such functions is as follows:

$$
\mathbf{B}(x, y, z)=\sum_{i=1}^{n} \sum_{j=1}^{m} \sum_{w=1}^{p} \mathbf{c}_{i, j, w} N_{i}(x) M_{j}(y) P_{w}(z),
$$

where $\mathbf{B}(x, y, z) \in \mathbb{R}^{3}$ is the magnetic field, $x, y, z \in \mathbb{R}$ are the parametrization variables, which we choose to be the Cartesian coordinates (Fig. 1). Further, $\left(\mathbf{c}_{i, j, w}\right)_{i, j, w=1}^{n, m, p} \in \mathbb{R}^{3}$ is the set of control points, and $N_{i}(x), M_{j}(y)$ and $P_{w}(z)$ are the basis functions of the B-Splines.

Furthermore, we assume - as is often the case in the literature [13], [20], [21] - to have field measurements from a grid of $s \times r \times u$ points. Consequently, it is possible to rearrange such measurements to obtain a matrix $\left(\mathbf{D}^{\star} \in \mathbb{R}^{s * r * u \times 3}\right)$ such that

$$
\mathbf{D}^{\star}=\left[\begin{array}{ccc}
D_{x}^{\star 1,1,1} & D_{y}^{\star 1,1,1} & D_{z}^{\star 1,1,1} \\
\vdots & \vdots & \vdots \\
D_{x}^{\star s, 1,1} & D_{y}^{\star s, 1,1} & D_{z}^{\star s, 1,1} \\
D_{x}^{\star 1,2,1} & D_{y}^{\star 1,2,1} & D_{z}^{\star 1,2,1} \\
\vdots & \vdots & \vdots \\
D_{x}^{\star s, r, 1} & D_{y}^{\star s, r, 1} & D_{z}^{\star s, r, 1} \\
D_{x}^{\star 1,1,2} & D_{y}^{\star 1,1,2} & D_{z}^{\star 1,1,2} \\
\vdots & \vdots & \vdots \\
D_{x}^{\star s, r, u} & D_{y}^{\star s, r, u} & D_{z}^{\star s, r, u}
\end{array}\right],
$$

where $D_{x, y, z}^{\star i, j, k}$ is the $x, y$ or $z$ component of the field for the measurement at $(i, j, k)$. Moreover, we define $\mathbf{D}$ as the matrix containing the value of the B-Spline at the coordinates of the measured points. If $\mathbf{D}$ is arranged as $\mathbf{D}^{\star}$, it follows from (6) that

$$
\mathbf{D}=\mathbf{Z C}
$$

with

$$
\mathbf{Z}=\left[\begin{array}{cccc}
N_{1}^{x_{1}} M_{1}^{y_{1}} P_{1}^{z_{1}} & \ldots & N_{n}^{x_{1}} M_{m}^{y_{1}} P_{p}^{z_{1}} \\
\vdots & \ddots & \vdots \\
N_{1}^{x_{s}} M_{1}^{y_{1}} P_{1}^{z_{1}} & \ddots & N_{n}^{x_{s}} M_{m}^{y_{1}} P_{p}^{z_{1}} \\
N_{1}^{x_{1}} M_{1}^{y_{2}} P_{1}^{z_{1}} & \ddots & N_{n}^{x_{1}} M_{m}^{y_{2}} P_{p}^{z_{1}} \\
\vdots & \ddots & \vdots \\
N_{1}^{x_{s}} M_{1}^{y_{r}} P_{1}^{z_{u}} & \ldots & N_{n}^{x_{s}} M_{m}^{y_{r}} P_{p}^{z_{u}}
\end{array}\right]
$$




$$
\mathbf{C}=\left[\begin{array}{ccc}
c_{x}^{1,1,1} & c_{y}^{1,1,1} & c_{z}^{1,1,1} \\
\vdots & \vdots & \vdots \\
c_{x}^{n, 1,1} & c_{y}^{n, 1,1} & c_{z}^{n, 1,1} \\
c_{x}^{1,2,1} & c_{y}^{1,2,1} & c_{z}^{1,2,1} \\
\vdots & \vdots & \vdots \\
c_{x}^{n, m, p} & c_{y}^{n, m, p} & c_{z}^{n, m, p}
\end{array}\right]
$$

where $\left(x_{i}, y_{j}, z_{i}\right)$ are the parametrized coordinates of the measured point, $\mathbf{C} \in \mathbb{R}^{n * m * p \times 3}$ is the matrix of control points associated to the $n * m * p$ basis functions of the tensor product B-Spline, and $N_{i}^{\alpha}=N_{i}(\alpha)$. Therefore, $\mathbf{Z} \in \mathbb{R}^{s * r * u \times n * m * p}$ maps the control points to the values of the function in the selected points.

Further, it is necessary to enforce Maxwell's equation for a quasi-static field measured outside of the electromagnet with no electrical disturbance [13]

$$
\begin{array}{r}
\nabla \cdot \mathbf{B}=\frac{\partial \mathbf{B}_{x}}{\partial x}+\frac{\partial \mathbf{B}_{y}}{\partial y}+\frac{\partial \mathbf{B}_{z}}{\partial z}=0 \\
\nabla \times \mathbf{B}=\left[\begin{array}{l}
\frac{\partial \mathbf{B}_{z}}{\partial z}-\frac{\partial \mathbf{B}_{y}}{\partial z} \\
\frac{\partial \mathbf{B}_{x}}{\partial z}-\frac{\partial \mathbf{B}_{z}}{\partial x} \\
\frac{\partial \mathbf{B}_{y}}{\partial x}-\frac{\partial \mathbf{B}_{x}}{\partial y}
\end{array}\right]=0
\end{array}
$$

where $\mathbf{B}=\mathbf{B}(x, y, z)$ for brevity and $\nabla$ is the gradient operator.

For this purpose, it is necessary to obtain the partial derivatives of the tricubic spline using a formal derivation of (6)

$$
\begin{aligned}
& \frac{\partial \mathbf{B}(x, y, z)}{\partial x}=\sum_{i=1}^{n} \sum_{j=1}^{m} \sum_{w=1}^{p} \mathbf{c}_{i, j, w} \dot{N}_{i}(x) M_{j}(y) P_{w}(z), \\
& \frac{\partial \mathbf{B}(x, y, z)}{\partial y}=\sum_{i=1}^{n} \sum_{j=1}^{m} \sum_{w=1}^{p} \mathbf{c}_{i, j, w} N_{i}(x) \dot{M}_{j}(y) P_{w}(z), \\
& \frac{\partial \mathbf{B}(x, y, z)}{\partial z}=\sum_{i=1}^{n} \sum_{j=1}^{m} \sum_{w=1}^{p} \mathbf{c}_{i, j, w} N_{i}(x) M_{j}(y) \dot{P}_{w}(z),
\end{aligned}
$$

where $\dot{N}_{i}(x)=\frac{\partial N_{i}(x)}{\partial x}, \dot{M}_{i}(y)=\frac{\partial M_{i}(y)}{\partial y}$, and $\dot{P}_{i}(z)=\frac{\partial P_{i}(z)}{\partial z}$. Finally, (13) can be substituted in (11) and (12) to obtain the analytical constraints of the tensor B-spline.

\section{Multi-Coil mapping With Static Disturbance}

The procedure presented in the previous section can be iterated to obtain a magnetic map for multi-coil systems affected by homogeneous or inhomogeneous disturbances. In point of fact, if the electromagnets have not reached magnetic saturation, the overall field in such systems can be computed as [13], [22]

$$
\mathbf{B}(\mathbf{p})=\sum_{i=1}^{n_{e}} \mathbf{B}_{i}(\mathbf{p})+\mathbf{B}_{d}(\mathbf{p})=\sum_{i=1}^{n_{e}} \widetilde{\mathbf{B}_{i}}(\mathbf{p}) I_{i}+\mathbf{B}_{d}(\mathbf{p}),
$$

where $n_{e}$ is the number of electromagnets, $\mathbf{p} \in \mathbb{R}^{3}$ is the position in which the field is evaluated, $\mathbf{B}_{i} \in \mathbb{R}^{3}$ is the field generated by the $i$-th electromagnet. Moreover, $\mathbf{B}_{d} \in \mathbb{R}^{3}$ is the field sourced from the disturbance, $\widetilde{\mathbf{B}_{i}} \in \mathbb{R}^{3}$ is the vector mapping currents to field of the $i$-th electromagnet, and $I_{i}$ is the current fed to such electromagnet.
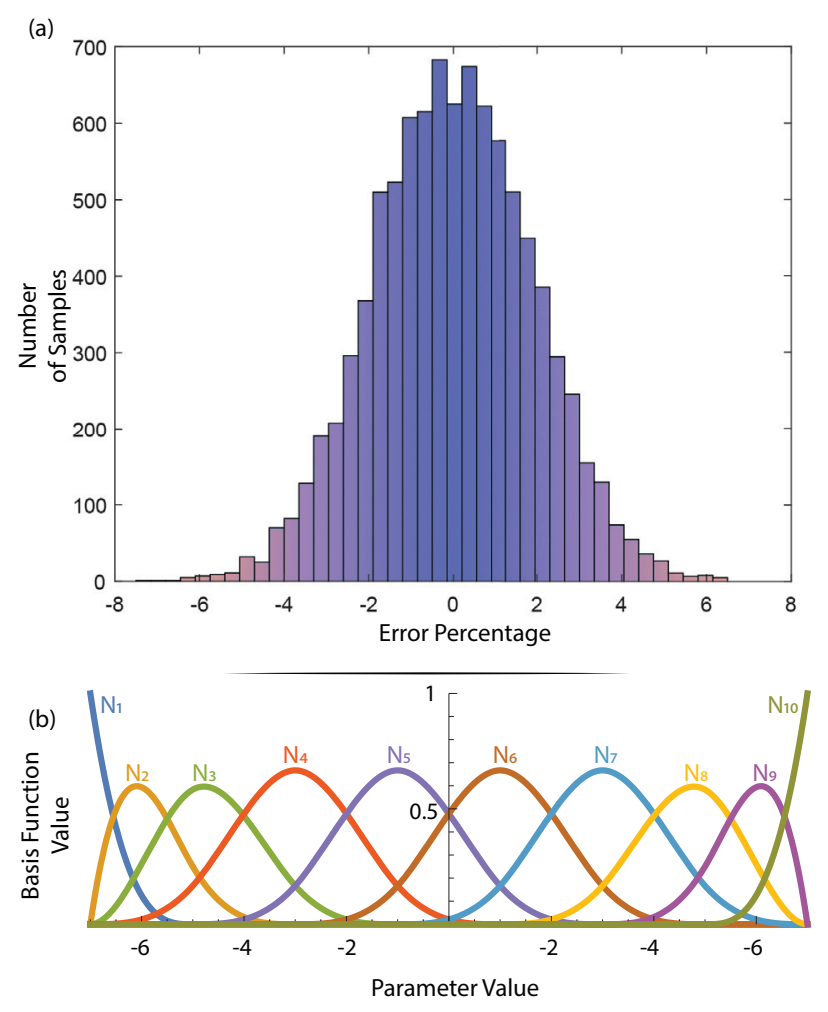

Fig. 2: Top (a): Histogram of relative error residuals for the mapping procedure. The procedure validated the technique with a coefficient of determination of 0.9884 , maximum relative error of $-7 \%$, and a standard deviation of residuals of $2.2 \%$. Bottom (b) Graphical representation of one of the used sets of cubic basis functions with a open uniform knot vector. The knots of the depicted basis functions were positioned on the grid of measured points (Fig. 1).

In order to calibrate the overall field we have to compute the tensor-product B-splines of the $n_{e} \widetilde{\mathbf{B}_{i}}$ and of $\mathbf{B}_{d}$. First, we measure the field when all the magnetic sources are fed no current. It is clear from (14) that this will correspond to a measurement of $\mathbf{B}_{d}$. Further, we iteratively measure the field as only one electromagnet is powered on. In such condition, the current-to-field map can be computed as

$$
\widetilde{\mathbf{B}_{i}}(\mathbf{p})=\frac{\mathbf{B}_{m}(\mathbf{p})-\mathbf{B}_{d}(\mathbf{p})}{I_{i}}, \quad I_{j}=0 \quad \forall j \neq i,
$$

where $\mathbf{B}_{m}(\mathbf{p})$ is the field measured at point $\mathbf{p} \in \mathbb{R}^{3}$. Further, it is worth noting that the measurements do not have to be collected in the same points, nor in the same number. In point of fact, after the first measurement the tensor-product B-spline map of $\mathbf{B}_{d}$ can be used in (15).

\section{MAPPING AND EXPERIMENTAL RESULTS}

The first step in the construction of a tensor product BSpline for magnetic mapping consists in the choice of knots and degrees. However, no combination of these variables is $a$ priori superior for magnetic mapping. Given the vast literature regarding the selection of B-spline knots and degrees, we will limit the discussion to a few considerations [23]-[26]: 


\section{1) Knots selection}

Every point in the B-Spline will belong to the convex-hull of the control points associated with the $d+1$ neighboring knots (See II). Consequently, selecting a number of knots that is significantly smaller than the number of measured points leads to a poor quality fit. Conversely, using significantly more knots than measured points may result in a map that is overfitted to the training data.

\section{2) Degree of the B-Spline}

An increase in the B-Spline degree generally offers a closer fit to the training data. However, high degree curves often result in overfitting and/or increases in the value of high-order derivatives, resulting in issues such as ringing. On the other hand, a B-Spline degree of at least two is necessary to ensure continuous gradients. We found third and fifth order B-Splines to yield the lowest errors. Nonetheless, the fifth order B-spline did present higher elongation and ringing than third order Bsplines, with an average increase of $83 \%$ in the Frobenius norm of the Hessian matrices [27].

\section{Fitting to data}

Given the knots and degrees of the B-Splines, $\mathbf{Z}^{\dagger}-$ the Moore-Penrose pseudo-inverse of $\mathbf{Z}$ - can be used to obtain a least square fit of the tensor product B-Spline to the measurements using $\mathbf{C}=\mathbf{Z}^{\dagger} \mathbf{D}^{\star}$ while enforcing (11) and (12).

However, as the number of knots increases, solving (11) and (12) analytically requires significant computational power. Alternatively, we use the following convex linearly-constrained quadratic approximation:

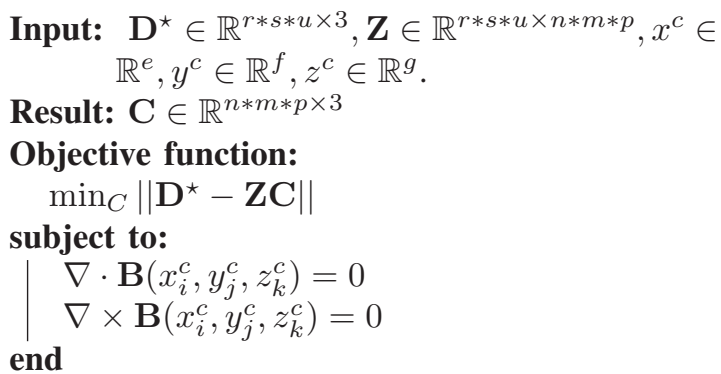

with $i=1, \ldots, e+1 ; j=1, \ldots, f+1 ; z=1, \ldots, g$. In this approximation, Maxwell's equations are only enforced on the grid of $e \times f \times g$ points. Nonetheless, as reported in Table I these can selected to have arbitrary small curl and divergence values in any point of the mapping function.

The presented technique is validated with the mapping of an electromagnetic testbed (Fig. 1) equipped with nine metal-core electromagnets affected by a static disturbance (Table I). A 3D grid of $8 \times 8 \times 8$ points is used as training set, while a grid of $7 \times 7 \times 7$ points is used for validation (Fig. 2). For improved accuracy, the data was collected using a calibrated three-axis teslameter (SENIS, Zurich, Switzerland) positioned by a 6-DOF robotic arm (UR5, Universal Robots, Odense, Denmark).

\section{Special Cases}

It should be noted that the current validation is performed on a cube of uniformly spaced measurements. Notwithstanding,
TABLE I: Summary of the experimental results. A non-uniform static disturbance with average value $1.1 \mathrm{mT}$ is introduced positioning a $30 \times 10 \varnothing \mathrm{mm}$ cylindrical N45 permanent magnet at a distance of 10 $\mathrm{cm}$, along the gravitational axis, from the center of the workspace. Open uniform knots vectors are used. Further, the boundary conditions are respected by defining a $d+1$ multiplicity for the first and last knots. The quadratic programs are solved using fmincon (MatLab, MathWorks, Natick, United States). Abbreviations are as follows: Number (\#), Coefficient of Determination $\left(\mathrm{R}^{2}\right)$, Adjusted (Adj.), Maximum (Max.), Divergence (Div.).

\begin{tabular}{ccccccc}
$d$ & \# of Knots & $\mathbf{R}^{2}$ & $\begin{array}{c}\text { Adj. } \\
\mathbf{R}^{2}\end{array}$ & $\begin{array}{c}\text { Max. } \\
\text { Error }\end{array}$ & $\begin{array}{c}\text { Max. } \\
\text { Curl } \\
{[\mu \mathrm{T} / \mathrm{m}]}\end{array}$ & $\begin{array}{c}\text { Max. } \\
\text { Div. } \\
{[\mu \mathrm{T} / \mathrm{m}]}\end{array}$ \\
$\mathbf{3}$ & $14 \times 14 \times 14$ & 0.9884 & 0.9883 & $7.00 \%$ & 15.24 & 21.74 \\
$\mathbf{3}$ & $19 \times 19 \times 19$ & 0.9587 & 0.9585 & $13.11 \%$ & 8.11 & 5.54 \\
\hline $\mathbf{5}$ & $14 \times 14 \times 14$ & 0.9712 & 0.9710 & $8.85 \%$ & 1.88 & 0.7 \\
\hline
\end{tabular}

this technique can also be applied to irregularly shaped workspaces using non-uniformly spaced measurements. For this purposes the following should be observed:

\section{1) Non-uniform measurements}

The technique as presented is already suitable for magnetic mapping using non-uniform measurements. However, the use of a non-uniform knots distribution with higher knot-density in volumes having higher density of measurement could yield an improved fit in such scenario.

\section{2) Irregularly shaped workspaces}

In such a scenario a lattice big enough to inscribe the irregular workspace has to be selected. It will be sufficient to then assign a multiplicity of $d+1$ to the knots on the edge of the irregular workspace. Any value can then be assigned to the control points outside of the area of interest.

\section{CONCLUSIONS}

We present a novel technique for precise model-free mapping of electromagnetic systems. Such spline-based technique is able to accurately map the generated field. Moreover, this technique is capable of addressing the presence of inhomogeneous static electromagnetic disturbances or asymmetric electromagnets, and has no lower or upper bound on the number of required measurements. Furthermore, a time-effective numerical approximation of the mapping procedure is also presented. The theoretical work is validated with the mapping of an electromagnetic system with nine metal-core electromagnets in the presence of a static electromagnetic disturbance. Overall, the presented work provides a flexible technique for the precise mapping of electromagnetic fields and gradients even in the presence of axial asymmetry or disturbances.

In future work we will analyze the performance this technique when mapping other electromagnetic setups. Further, a quantitative comparison of time efficiency of techniques for the mapping of electromagnetic fields will be performed. Finally, we will investigate techniques aimed at increasing the time effectiveness of the presented procedure.

\section{ACKNOWLEDGMENTS}

The authors would like to thank Federico Califano (Department of Electrical, Electronic and Information Engineering, 
University of Bologna, Italy) for the numerous insightful discussions and helpful suggestions that have led to the presented work.

This research has received funding from the European Research Council (ERC) under the European Union's Horizon 2020 Research and Innovation programme (Grant Agreement \#638428 - project ROBOTAR: Robot-Assisted Flexible Needle Steering for Targeted Delivery of Magnetic Agents).

\section{REFERENCES}

[1] A. G. Banerjee, S. Chowdhury, S. K. Gupta, and W. Losert, "Survey on indirect optical manipulation of cells, nucleic acids, and motor proteins,' Journal of Biomedical Optics, vol. 16, no. 5, p. 051302, 2011.

[2] S. Martel, "Magnetic navigation control of microagents in the vascular network: Challenges and strategies for endovascular magnetic navigation control of microscale drug delivery carriers," IEEE Control Systems, vol. 33, no. 6, pp. 119-134, 2013.

[3] S. Chowdhury, W. Jing, and D. J. Cappelleri, "Controlling multiple microrobots: recent progress and future challenges," Journal of MicroBio Robotics, vol. 10, no. 1-4, pp. 1-11, 2015.

[4] F. Ongaro, S. Scheggi, C. Yoon, F. van den Brink, S. H. Oh, D. H. Gracias, and S. Misra, "Autonomous planning and control of soft untethered grippers in unstructured environments," Journal of Micro-Bio Robotics, pp. 1-8, 2016.

[5] F. Ongaro, C. Yoon, F. van den Brink, M. Abayazid, S. H. Oh, D. H. Gracias, and S. Misra, "Control of untethered soft grippers for pick-andplace tasks," in IEEE International Conference on Biomedical Robotics and Biomechatronics (BioRob), pp. 299-304, 2016.

[6] H. Ceylan, J. Giltinan, K. Kozielski, and M. Sitti, "Mobile microrobots for bioengineering applications," Lab on a Chip, vol. 17, no. 10, pp. 1705-1724, 2017.

[7] P. Ryan and E. Diller, "Magnetic actuation for full dexterity microrobotic control using rotating permanent magnets," IEEE Transactions on Robotics, vol. 33, no. 6, pp. 1398-1409, 2017.

[8] Z. Yan, M. Han, Y. Shi, A. Badea, Y. Yang, A. Kulkarni, E. Hanson, M. E. Kandel, X. Wen, F. Zhang et al., "Three-dimensional mesostructures as high-temperature growth templates, electronic cellular scaffolds, and self-propelled microrobots," Proceedings of the National Academy of Sciences, vol. 114, no. 45, pp. E9455-E9464, 2017.

[9] D. Rus and M. T. Tolley, "Design, fabrication and control of origami robots," Nature Reviews Materials, p. 1, 2018.

[10] J. Rahmer, C. Stehning, and B. Gleich, "Remote magnetic actuation using a clinical scale system," PloS one, vol. 13, no. 3, p. e0193546, 2018 .

[20] F. Ongaro, C. Pacchierotti, C. Yoon, D. Prattichizzo, D. H. Gracias, and S. Misra, "Evaluation of an electromagnetic system with haptic feedback for control of untethered, soft grippers affected by disturbances," in Proceedings of the IEEE International Conference on Biomedical Robotics and Biomechatronics (BioRob), pp. 900-905, 2016.
[11] A. J. Petruska and B. J. Nelson, "Minimum bounds on the number of electromagnets required for remote magnetic manipulation," IEEE Transactions on Robotics, vol. 31, no. 3, pp. 714-722, 2015.

[12] A. Denasi and S. Misra, "Independent and leader-follower control for two magnetic micro-agents," IEEE Robotics and Automation Letters, vol. 3, no. 1, pp. 218-225, 2018.

[13] A. J. Petruska, J. Edelmann, and B. J. Nelson, "Model-based calibration for magnetic manipulation,' IEEE Transactions on Magnetics, vol. 53, no. 7, pp. 1-6, 2017.

[14] D. F. Rogers, An introduction to NURBS: with historical perspective. Elsevier, 2000.

[15] T. Martin, E. Cohen, and R. M. Kirby, "Volumetric parameterization and trivariate b-spline fitting using harmonic functions," Computer aided geometric design, vol. 26, no. 6, pp. 648-664, 2009.

[16] K. Wang, X. Li, B. Li, H. Xu, and H. Qin, "Restricted trivariate polycube splines for volumetric data modeling," IEEE Transactions on Visualization and Computer Graphics, vol. 18, no. 5, pp. 703-716, 2012.

[17] L. Biagiotti, F. Califano, and C. Melchiorri, "Repetitive control of nonminimum phase systems along b-spline trajectories," in Decision and Control (CDC), 2016 IEEE 55th Conference on, pp. 5496-5501. IEEE, 2016.

[18] E. Cohen, R. F. Riesenfeld, and G. Elber, Geometric modeling with splines: an introduction. AK Peters/CRC Press, 2001.

[19] L. Piegl and W. Tiller, The NURBS book. Springer Science \& Business Media, 2012.

[21] J. Sikorski, I. Dawson, A. Denasi, E. E. Hekman, and S. Misra, "Introducing bigmag - a novel system for 3d magnetic actuation of flexible surgical manipulators," in IEEE International Conference on Robotics and Automation (ICRA), pp. 3594-3599, 2017.

[22] F. Ongaro, S. Scheggi, A. Ghosh, A. Denasi, D. H. Gracias, and S. Misra, "Design, characterization and control of thermally-responsive and magnetically-actuated micro-grippers at the air-water interface," PloS one, vol. 12, no. 12, p. e0187441, 2017.

[23] V. Braibant and C. Fleury, "Shape optimal design using b-splines," Computer Methods in Applied Mechanics and Engineering, vol. 44, no. 3, pp. 247-267, 1984

[24] D. Ruppert, "Selecting the number of knots for penalized splines," Journal of computational and graphical statistics, vol. 11, no. 4, pp. 735-757, 2002.

[25] H. Park and J.-H. Lee, "B-spline curve fitting based on adaptive curve refinement using dominant points," Computer-Aided Design, vol. 39, no. 6, pp. 439-451, 2007.

[26] Y. Yuan, N. Chen, and S. Zhou, "Adaptive b-spline knot selection using multi-resolution basis set," Iie Transactions, vol. 45, no. 12, pp. 1263$1277,2013$.

[27] W. C. Thacker, "The role of the hessian matrix in fitting models to measurements," Journal of Geophysical Research: Oceans, vol. 94, no. C5, pp. 6177-6196, 1989. 